\title{
Research on the Multiroute Probit-Based Public Transit Assignment Model Based on Bus Stop
}

\author{
Zhenyu Mei, ${ }^{1}$ Dianhai Wang, ${ }^{1}$ Fujian Wang, ${ }^{1}$ Jun Chen, ${ }^{2}$ \\ and Wei Wang ${ }^{2}$ \\ ${ }^{1}$ Department of Civil Engineering, Zhejiang University, Hangzhou 310058, China \\ ${ }^{2}$ Department of Transportation, Southeast University, Nanjing 210098, China
}

Correspondence should be addressed to Zhenyu Mei, meizhenyu@zju.edu.cn

Received 25 February 2012; Revised 31 May 2012; Accepted 1 June 2012

Academic Editor: Gerhard-Wilhelm Weber

Copyright (C) 2012 Zhenyu Mei et al. This is an open access article distributed under the Creative Commons Attribution License, which permits unrestricted use, distribution, and reproduction in any medium, provided the original work is properly cited.

\begin{abstract}
A public transit network differs from a general road network. The passenger flow of bus stops and the limited capacity of buses have a greater effect than road traffic flow on the running time of buses. As a result, conventional public transit assignment models that adopt the econometric road network path concept have numerous limitations. Based on the analysis, the generalized bus trip time chain is analyzed, and the concept of a congestion function is proposed to describe the relationship between trip resistance and flow in the current paper. On the premise of this study, the transit network resistance function is formed and the multiroute probit-based loading model is established. With using STOCH or Dial's algorithm, the process of distribution is proposed. Finally, the model is applied to the transit network assignment of Deqing Town in Zhejiang Province. The result indicates that the model can be applied to practical operations with high-precision results.
\end{abstract}

\section{Introduction}

Public transit passenger flow distribution is the core of public transit network planning. A great deal of research has been conducted on this subject over the past 30 years. Daganzo and Sheffi proposed the probit-based distribution model [1, 2], but this method cannot accommodate the demands of a large-scale network. The greatest achievement within the twentieth century in terms of the public transit assignment technique is the trip strategy theory that was put forward in the 1980s [3]. The trip strategy theory not only reflects the characteristics of the passengers' trip but also adapts to the public transit network by improving the equilibrium assignment model of motor vehicles. De Cea and Fernandez define attraction route collection [4] and propose a new assignment algorithm based on frequency to further analyze public transit trip characteristics. Wu et al. propose 
an assignment algorithm that is based on a new theory and is integrated with super path [5]. However, these models disregard the capacity constraints of vehicles. Thus, the distribution results exceed the route capacity [6]. Moreover, even when the capacity constraints of vehicles are considered, these models disregard the particularity of the public transit network, adopt the path concept of common road network, and oversimplify the passengers' waiting time in bus stops $[7,8]$. Therefore, these models are applicable only to small-scale public transit networks. These models also presume that congestion is not a possible condition, that is, the models presume that all bus routes have sufficient capacity to satisfy all the demand. However, this hypothesis on a condition without congestion only exists in theoretical research and thus cannot be applied to a practical public transit network.

The conventional models of public transit assignment adopt the road impedance function to reflect the relationship between traffic flow and road impedance, which is called road impedance renovation. However, the route congestion effects of the public transit network are primarily reflected in bus stops, that is, the trip time between the two stops has little correlation with the variety of the route flow [9-12]. Therefore, the relationship between the impedance of the trip path and passenger flow is represented by the relationship between path impedance and stop congestion effects, called stop-based impedance renovation. The congestion effect of the public transit system reflects the delay resulting from the capacity constraints of vehicles [13]. Schmöcker et al. present an approach to dynamic frequencybased transit assignment based on the line capacity constraints. The paper establishes the assignment model by introduction of a "fail-to-board" probability as in some circumstances passengers are not able to board the first service arriving due to overcrowding [14] . Nuzzolo et al. present a schedule-based dynamic assignment model for transit networks, which takes into account congestion through explicit vehicle capacity constraints [15] . But the detailed choosing behavior of passengers and bus trip time chain are not analyzed.

The detailed choosing behavior of passengers then progresses as follows: if the first expected bus is overly crowded, the next bus or another route will be selected. Based on this premise, the congestion function is proposed. Integrating with the study of the public transit trip time chain, the multiroute probit-based loading model is established, and the algorithm is analyzed.

To address the aforementioned issues, the present study focuses on the following:

(1) analyze bus trip time chain and propose congestion function based on bus stop;

(2) establish multiroute probit-based loading model and analyze the algorithm;

(3) Investigate the operational performance of the model through five bus routes assignments of Deqing Town in Zhejiang Province.

\section{Public Transit Network Impedance}

The most important step in public transit assignment is to confirm the selected path's traffic impedance. Public transit network impedance refers to the comprehensive expense guideline for trip time (including out-vehicle time), fees, and convenience (transfer rate) in a given public transit network, which is the rationale behind passenger selection of public transport.

As shown in Figure 1, the bus route section and walking route section are called the bus and walking paths. Public transit network impedance should reflect the behavior and real psychological characteristics of passengers in considering time and expense factors. These 


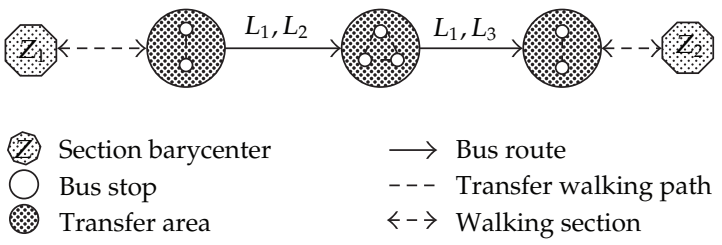

Figure 1: Sketch map of generalized public transit network between sections $Z_{1}$ and $Z_{2}$.

time and expense factors can be denoted by "fee," also called generalized fee [16]. Bus trip impedance adopts a common array form and can be expressed as follows:

$$
A_{i}=\left(i, f\left(T, L, \bar{v}_{i}, p\right)\right)
$$

where $i$ denotes the number of bus paths. $f\left(T, L, \bar{v}_{i}, p\right)$ is the generalized fee function, among which,

$$
f\left(T, L, \bar{v}_{i}, p\right)=C \cdot T_{i}+\psi\left(L_{i}, \bar{v}_{i}, p_{i}\right),
$$

where $C$ is the time value, adopting the corresponding year's national income per person (Yuan $/ \mathrm{h}) ; T_{i}$ refers to the total duration of the bus trip; $\psi(\cdot)$ is the fee function of the bus trip, relating with trip distance $L_{i}(\mathrm{~km})$, average bus speed $\bar{v}_{i}(\mathrm{~km} / \mathrm{h})$, and fare $p_{i}(Y u a n / \mathrm{km})$. The bus trip time chain and congesting function are introduced to reflect public transit network impedance in this paper.

\subsection{Bus Trip Time Chain Analysis}

In the course of the bus trip, generalized trip time $T$ includes the time consumed walking to the bus stop and walking home, waiting for the bus, trip time in the bus, and the transition time. All different parts constitute an integrated trip time chain.

Given that different parts of the bus trip chain have different values, $T$ does not simply denote the sum of the values of all parts. With the use of fee, the different choosing actions in different situations during the trip can be more accurately analyzed. Hence, the total trip time $T$ can be expressed by the following function:

$$
T=\sum_{i=1}^{3} \alpha_{i} t^{i}+\sum_{e=1}^{n} n \alpha_{e} t^{e}
$$

where $t^{i}(i=1,2,3)$ refers to the time consumed walking to the bus stop and walking home, waiting for the bus, and trip time in the bus; $n$ denotes the number of transitions; $\alpha$ is the time factor; $t^{e}$ is the total transition time (including the waiting time and walking time when changing routes).

Walking time $t_{1}$ correlates with walking distance $D_{1}$ and average walking speed $\bar{v}_{1}$. Waiting time in the bus stop $t_{2}$ is determined by service frequency $F$. Trip time in bus $t_{3}$ is determined by bus route length $D_{3}$ and running speed $\bar{v}_{3}$. Transition time correlates with the 


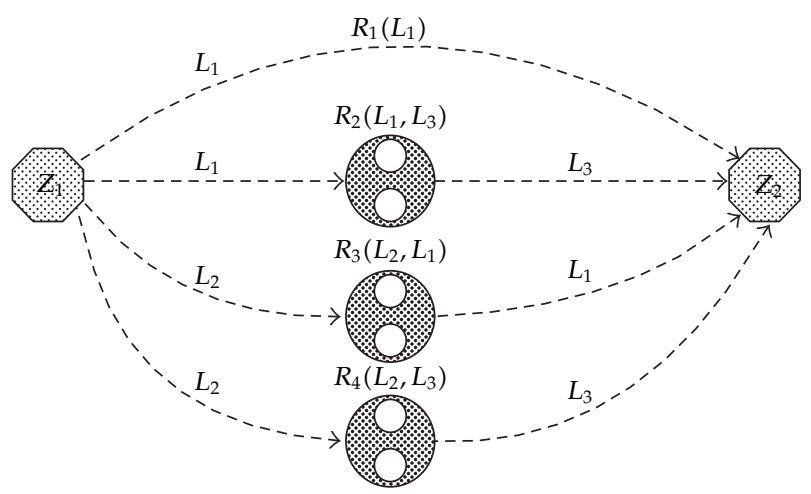

Figure 2: Sketch map of bus trip route $L$ between sections $Z_{1}$ and $Z_{2}$.

distance of the bus stops $D_{4}$ and the waiting time in bus stop $t_{2}$. Therefore, bus trip time can be denoted by the following formula:

$$
T=\alpha_{1} \frac{f_{w} D_{1}}{\bar{v}_{1}}+\alpha_{2} \frac{f_{a}}{F}+\alpha_{3}\left(\frac{D_{3}}{\bar{v}_{3}}+t_{3}^{\prime}\right)+n \alpha^{e}\left(\frac{f_{w} D_{4}}{\bar{v}_{1}}+\frac{f_{a}}{F}\right)
$$

where $f_{w}$ is the nonlinear coefficient, $f_{a}$ is the waiting time revising coefficient, and $t_{3}^{\prime}$ is the bus running time revising coefficient.

\subsection{Network Impedance Revision Based on Bus Stop}

The bus route $L$ between sections $Z_{1}$ and $Z_{2}$ is analyzed based on Figure 2 .

In reviewing the single bus route $L_{i}$, analyzing the sectional passenger flow $V_{l}^{s}$ of the $S$ section of bus route $L$ is the principal step in confirming the congestion function. Sectional passenger flow can be expressed by the following formula:

$$
V_{l}^{s}=\sum_{l} \sum_{s} \delta_{s l i} \cdot h_{i}
$$

where $V_{l}^{S}$ is the sectional passenger flow of the $S$ section of bus route $L, h_{i}$ is the path $i$ flow, and $\delta_{s l i}$ is the coefficient assuming that the $S$ section of bus route $L$ belongs to path $i$. If $S$ belongs to $i, i=1$; otherwise, $i=0$.

Based on the analysis of the sectional passenger flow $V_{l}^{s}$, the congestion function $\psi_{s}\left(V_{l}^{s} / K_{l}\right)$ is introduced to denote network impedance. When passengers are waiting for a bus in the bus stop and/or the bus route demands exceed the buses' capacity, congestion will occur in the bus. In this way, one portion of passengers will miss the bus or be unwilling to board the crowded vehicle. This condition will also facilitate the queue phenomena in the bus stop. Along with the increase in congestion, passengers will choose an alternate bus route with a lower speed, larger service interval, and detours instead of the crowded route after considering the degree of congestion, discomfort, and riding time. Therefore, the first corresponding factor can be expressed by the passengers' delay attributed to the limited 
capacity of the bus. This factor correlates with flow and can be expressed in the form of the Bureau of Public Roads formula [17]:

$$
\psi_{s}\left(\frac{V_{l}^{s}}{K_{l}}\right)=w_{w} \cdot\left(\frac{V_{l}^{s}}{K_{l}}\right)^{\beta}+w_{i}^{\prime} \cdot\left(\frac{V_{l}^{s}}{K_{l}}\right)^{\rho},
$$

where $w_{w}, w_{i}^{\prime}, \beta$, and $\rho$ are revising factors; $K_{l}$ is the maximal practical carrying capacity. Considering that the basic carrying capacity is affected by both stop berth capacity and repeated route, $K_{l}$ can be denoted by following formula:

$$
K_{l}=\gamma \cdot C_{x} \cdot \frac{f\left(X_{c}\right)}{T_{l}}
$$

where $C_{x}$ is the capacity of different vehicle types, commonly denoting either a middle autobus, single bus, or articulated buses with carrying capacities of 26, 72, and 129 persons, respectively. $T_{l}$ is the service interval in bus route $L_{i}(\mathrm{~min}) . \gamma$ is the loading rate of the route, assuming a rate of 0.85 during peak hours and 0.6 in flat hours [18]. $\gamma$ can also be valued according to the idiographic situation of each city. $X_{c}$ is the maximal number of repeat routes. $\varphi\left(X_{c}\right)$ is the route repeat influence coefficient, which, when $X_{c}=0$ or 1 , assumes the value of 1 ; when $X_{c}=2$ or 3, assumes the value of 0.85 ; when $X_{c} \geq 4$, assumes the value of 0.7.

Therefore, considering single route $L_{i}$, the route impedance can be denoted according to the definition of the generalized fee function:

$$
f\left(T, L, \bar{v}_{i}, P\right)= \begin{cases}C \cdot\left(T+\psi_{s}\left(\frac{V_{l}^{s}}{K_{l}}\right)\right)+\frac{L \cdot p}{100} & \text { Counting distance fare rule } \\ C \cdot\left(T+\psi_{s}\left(\frac{V_{l}^{s}}{K_{l}}\right)\right)+n \cdot p^{\prime} & \text { Single fare rule } \\ V_{l}^{s}=\sum_{l} \sum_{s} \delta_{s l i} \cdot h_{i} & \\ \psi_{s}\left(\frac{V_{l}^{s}}{K_{l}}\right)=w_{w} \cdot\left(\frac{V_{l}^{s}}{K_{l}}\right)^{\beta}+w_{i}^{\prime} \cdot\left(\frac{V_{l}^{s}}{K_{l}}\right)^{\rho} & \\ K_{l}=\frac{\gamma \cdot C_{x} \cdot \varphi\left(X_{c}\right)}{T_{l}} .\end{cases}
$$

\section{Multiroute Probit-Based Loading Model and Algorithm}

\subsection{Model Establishment}

The selection behavior of passengers is a variable factor. Passengers typically prefer the optimal route in terms of service frequency, service quality, and service costs. Considering the vehicle and passenger arrival distribution and economical situation based on probability, the route with the higher service frequency can attract more passengers. On the other hand, the 


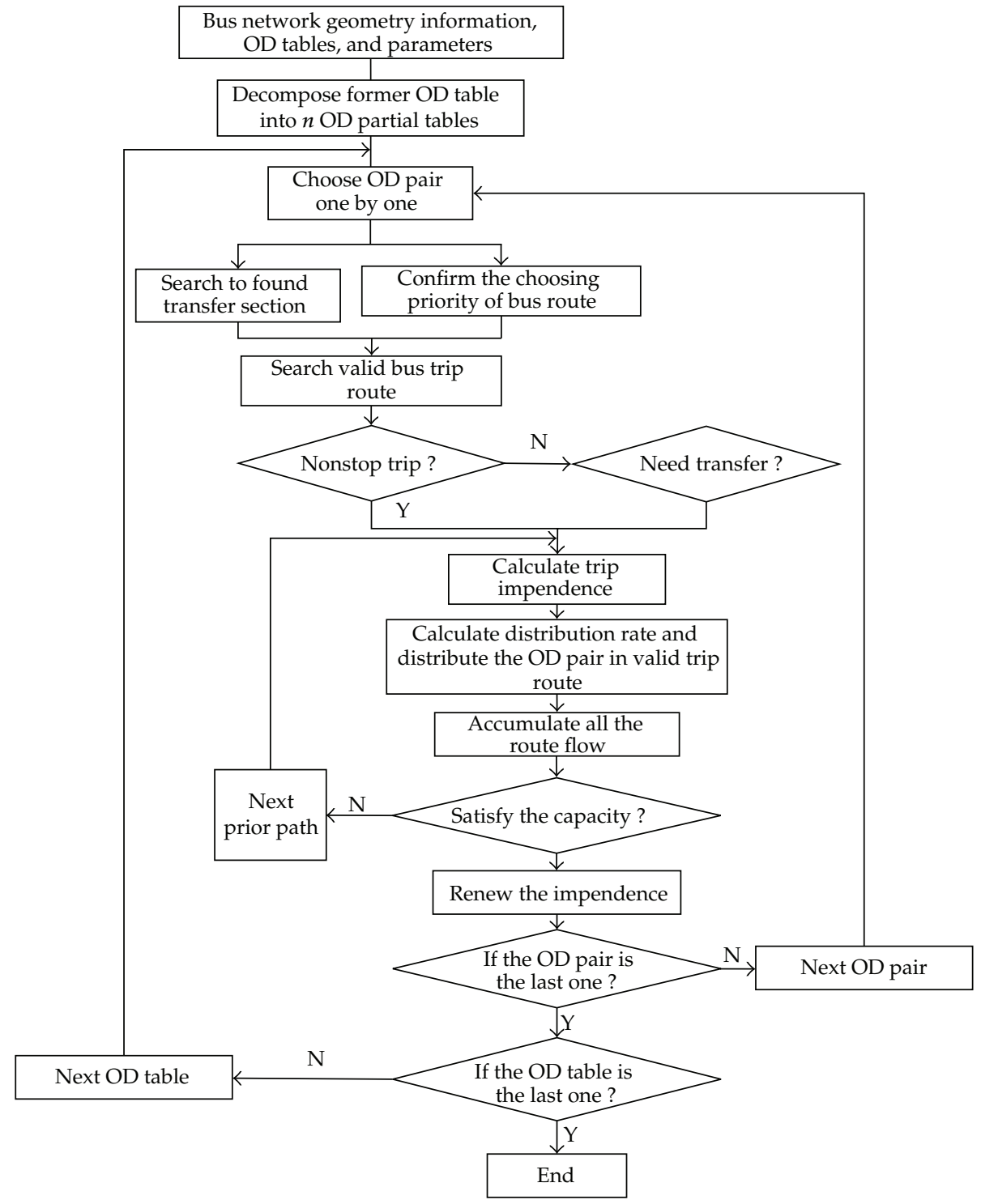

Figure 3: Multi-route probit-based loading model flow chart.

degree of congestion in the vehicle affects the passengers' choice of trip route. Thus, the user (passenger) equilibrium concept in the bus route differs from the user (vehicle) equilibrium concept. The latter reflects the equilibrium in travel time, given that travel time varies with the degree of road congestion. The former reflects the equilibrium of congestion degree in the vehicle aside from the equilibrium of travel time, given that the degree of congestion has less influence on travel time.

The multiroute probit-based loading model is proposed based on the following premises: assuming that a group of passengers is sent out from point of origin $r$ to destination $d$, multiroutes exist from $r$ to $d$. Given the passengers' lack of familiarity with the circumstances of the network and the fact that some factors are difficult to quantify, the 
passengers' estimation of path impedance is the random variable. The distribution model is utilized to calculate the number of passengers choosing each path based on the path estimation distribution function. following:

The chosen bus multiroute probit-based distribution model is constructed in the

$$
\begin{gathered}
P(k, i, j)=\frac{\exp \left(-a f_{k}\left(T, L, \bar{v}_{i}, p\right) / \bar{F}\right)}{\sum_{h=1}^{m} \exp \left(-a f_{h}\left(T, L, \bar{v}_{i}, p\right) / \bar{F}\right)}, \\
V(k, i, j)=V(i, j) \cdot P(k, i, j),
\end{gathered}
$$

where $P(k, i, j)$ is the distribution rate of public transit $\operatorname{OD} V(i, j)$ in the $k$ valid bus trip route. $f_{k}\left(T, L, \bar{v}_{i}, p\right)$ denotes the traffic impedance of the $k$ valid bus trip route. $\bar{F}$ is the average traffic impedance of each valid bus trip route. $a$ is the distribution parameter. $m$ denotes the number of valid bus trip routes. $V(k, i, j)$ is the distribution of public transit $\operatorname{OD} V(i, j)$ in the $k$ valid bus trip route.

\subsection{Multiroute Probit-Based Loading Algorithm}

In the process of distribution, the capacity constrained distribution model of capacity constrained-increment loading is integrated as a contributing factor. The former OD table is initially decomposed into K OD tables, and the K OD tables are then distributed successively using the multiroute probit-based loading model. When the distribution of each OD table is completed, the route impedance is calculated by adding trip time, fee, and flux. Afterwards, the distribution of the next OD table is performed until all OD tables are distributed, as shown in Figure 3. Incremental assignment solves the equilibrium model. Although incremental assignment typically does not produce equilibrium flow, it can get generate a close result to [19]. Assignment times can be derived using the following function:

$$
\begin{gathered}
{\left[q_{r s}^{k}\right]_{n \times n}=\left[P_{k} \cdot q_{r s}\right]_{n \times n^{\prime}}} \\
\sum_{k=1}^{K} P_{k}=1, \\
K=\left|\frac{C_{\max }}{C_{\min }}\right|, \\
P_{k}=\frac{K}{\sum_{i=1}^{K} i}=\frac{2 k}{(1+K) K^{\prime}}
\end{gathered}
$$

where $C_{\max }$ is the maximal capacity of a public transit network, $C_{\min }$ is the minimum capacity of the network, $\left[q_{r s}\right]_{n \times n}$ is the distributed OD in $k$ distribution, and $P_{k}$ denotes the distribution scale of $k$.

After the calculations, little difference is found between the calculated and experience values. Moreover, STOCH or Dial's algorithm can be effectively used to confirm all paths. STOCH algorithm or Dial's method can efficiently implement a probit-based choice model at 


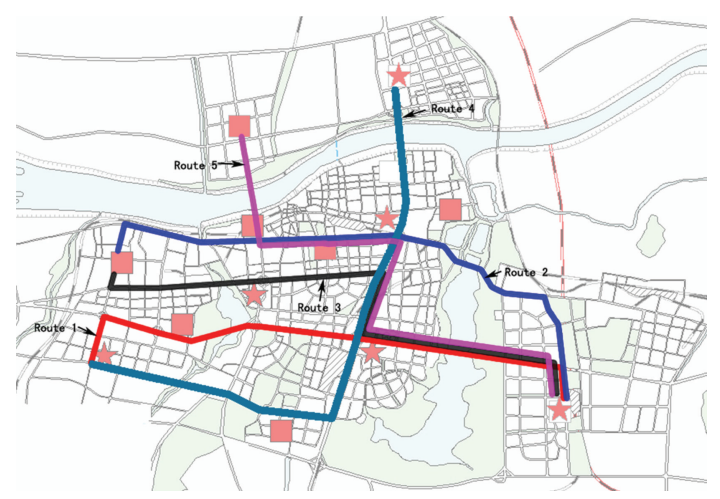

Figure 4: Sketch map of computation example.

the network level. The procedure assigns choice probabilities (or traffic flows) to reasonable paths connecting each OD pairs. The detail of STOCH algorithm or Dial's method can be found in [20]. This algorithm only considers the valid path comprising the section far from the trip origin and close to the trip destination. The OD is then assigned between the two points, and each section's distribution value is confirmed after the course of forward pass and backward pass in the network.

\section{Model Application}

This paper takes several parts of the urban area of Deqing Town in Zhejiang Province as an example to investigate the operational performance of the model. The sketch map and the five bus routes are shown in Figure 4. The Deqing public transit passenger information database is established on the basis of data obtained from the investigation.

Take the Route 1 bus passenger flow as an example to explain the model application. Route 1 has the length $10.2 \mathrm{~km}$, with a total of 17 stops, respectively, for the Guangming Road, Shishan Road, Lijin Hotel, Xixin Bridge, Ruijin Hospital, Southern Mall, CooperationBank, Dianzi Village, Telecom Building, Lanling Hotel, Pudong New Village, Qinliang Village, Nanping Street, Zhiyuan Road, Changhong Street, Changan Road, and Qianqiu Street. The average service frequency is every five minutes. The surveyed public transit OD data between 7:00 AM and 8:00 AM on April/10/2011, is shown in Table 1. The comparison among the assignments results and field data is shown in Table 2 and Figure 5.

From the comparisons shown in Table 2 and Figure 5, we can find the following.

(1) As the section flow increases, the assignments results will fluctuate within a certain range from the filed survey data, which is more or less related to the stochastic nature of public transit flow. However, the general tendencies of field data and calculation results are all the same, showing that assignments results can reflect the change of the section flow.

(2) When the section flow is between bus stops 10 and 11, the section flow has the maximal value and assignments results also get the maximal value. The assignments results are good representative of actual filed data.

Maximal route section flow is an effective index to evaluate the OD assignment results. After loading the OD table onto the public transit network, the maximal route section flow 
Table 1: The surveyed public transit OD data based on stops of Route 1.

\begin{tabular}{cccccccccccccccccc}
\hline OD & 1 & 2 & 3 & 4 & 5 & 6 & 7 & 8 & 9 & 10 & 11 & 12 & 13 & 14 & 15 & 16 & 17 \\
\hline 1 & 0 & 0 & 1 & 2 & 1 & 1 & 1 & 0 & 1 & 0 & 0 & 0 & 0 & 0 & 1 & 0 & 0 \\
2 & 0 & 0 & 3 & 3 & 2 & 4 & 1 & 2 & 2 & 1 & 1 & 2 & 1 & 1 & 1 & 0 & 0 \\
3 & 0 & 0 & 2 & 2 & 6 & 5 & 11 & 3 & 4 & 2 & 2 & 5 & 4 & 3 & 2 & 0 & 1 \\
4 & 0 & 0 & 0 & 0 & 2 & 1 & 3 & 1 & 2 & 1 & 2 & 4 & 4 & 3 & 3 & 1 & 1 \\
5 & 0 & 0 & 0 & 0 & 0 & 0 & 2 & 1 & 1 & 1 & 1 & 3 & 3 & 4 & 3 & 1 & 1 \\
6 & 0 & 0 & 0 & 0 & 0 & 0 & 0 & 0 & 1 & 1 & 1 & 4 & 5 & 6 & 6 & 2 & 3 \\
7 & 0 & 0 & 0 & 0 & 0 & 0 & 0 & 0 & 1 & 1 & 2 & 8 & 12 & 14 & 14 & 7 & 6 \\
8 & 0 & 0 & 0 & 0 & 0 & 0 & 0 & 0 & 0 & 0 & 1 & 3 & 3 & 7 & 7 & 5 & 7 \\
9 & 0 & 0 & 0 & 0 & 0 & 0 & 0 & 0 & 0 & 0 & 0 & 1 & 3 & 4 & 7 & 4 & 7 \\
10 & 0 & 0 & 0 & 0 & 0 & 0 & 0 & 0 & 0 & 0 & 0 & 1 & 3 & 6 & 10 & 7 & 12 \\
11 & 0 & 0 & 0 & 0 & 0 & 0 & 0 & 0 & 0 & 0 & 0 & 0 & 0 & 1 & 2 & 2 & 4 \\
12 & 0 & 0 & 0 & 0 & 0 & 0 & 0 & 0 & 0 & 0 & 0 & 0 & 1 & 2 & 3 & 2 & 7 \\
13 & 0 & 0 & 0 & 0 & 0 & 0 & 0 & 0 & 0 & 0 & 0 & 0 & 0 & 0 & 3 & 2 & 3 \\
14 & 0 & 0 & 0 & 0 & 0 & 0 & 0 & 0 & 0 & 0 & 0 & 0 & 0 & 0 & 2 & 1 & 5 \\
15 & 0 & 0 & 0 & 0 & 0 & 0 & 0 & 0 & 0 & 0 & 0 & 0 & 0 & 0 & 0 & 0 & 0 \\
16 & 0 & 0 & 0 & 0 & 0 & 0 & 0 & 0 & 0 & 0 & 0 & 0 & 0 & 0 & 0 & 0 & 0 \\
17 & 0 & 0 & 0 & 0 & 0 & 0 & 0 & 0 & 0 & 0 & 0 & 0 & 0 & 0 & 0 & 0 & 0 \\
\hline
\end{tabular}

Table 2: The route section flow of assignments results and surveyed data of Route 1.

\begin{tabular}{lccc}
\hline Stop code & Stop name & Route section flow of assignments results & Surveyed section flow \\
\hline 1 & Guangming Road & 1 & 1 \\
2 & Shishan Road & 5 & 6 \\
3 & Lijin Hotel & 13 & 16 \\
4 & Xixin Bridge & 17 & 19 \\
5 & Ruijin Hospital & 19 & 21 \\
6 & Southern Mall & 22 & 24 \\
7 & Cooperation Bank & 29 & 28 \\
8 & Dianzi Village & 33 & 34 \\
9 & Telecom Building & 35 & 36 \\
10 & Lanling Hotel & 40 & 41 \\
11 & Pudong New Village & 40 & 42 \\
12 & Qinliag Village & 37 & 38 \\
13 & Nanping Street & 32 & 30 \\
14 & Zhiyuan Road & 25 & 26 \\
15 & Changhong Street & 15 & 14 \\
16 & Changan Road & 9 & 8 \\
\hline
\end{tabular}

assignments results can be obtained. The comparison of maximal route section flow among the assignments results and field survey data is shown in Table 3.

According to the field survey data and computation results, the model is capable of estimating the maximum route section flow based on the OD table. The percentage of the difference between the maximal route section flow of assignments results and actual section route flow is less than $10 \%$. 


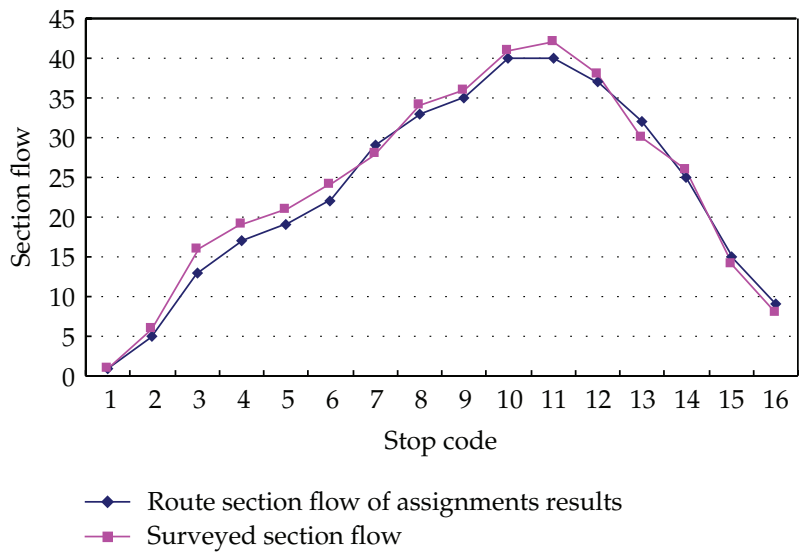

Figure 5: Comparison among the assignments results and field data of Route 1.

Table 3: Comparison of maximal route section flow.

\begin{tabular}{lccc}
\hline Route & $\begin{array}{c}\text { Maximal route section flow of } \\
\text { assignments results }\end{array}$ & $\begin{array}{c}\text { Actual maximal section } \\
\text { route flow }\end{array}$ & Discrepancy percentage \\
\hline 1 & 7379 & 7580 & $2.7 \%$ \\
2 & 17048 & 18344 & $7.1 \%$ \\
3 & 30220 & 32821 & $7.9 \%$ \\
4 & 15440 & 14374 & $7.4 \%$ \\
5 & 37880 & 36743 & $3.1 \%$ \\
\hline
\end{tabular}

\section{Conclusion}

This paper highlights the limitations of the equilibrium assignment model in practical applications based on an analysis of the conventional equilibrium assignment model of the public transit network. The multiroute probit-based distribution model is established, and the multiroute capacity-constrained nonequilibrium algorithm is analyzed. The model was applied to the transit network assignment of Deqing with satisfactory results.

\section{Acknowledgments}

The current work is supported by the Zhejiang Provincial Natural Science Foundation of China (no. LY12E08020) and the National High-Tech Research and Development Program (863 Program) (no. 2011AA110302).

\section{References}

[1] Y. Sheffi, "A note on the turn and arrival likelihood algorithms of traffic assignments," Transportation Research Part B, vol. 13, no. 2, pp. 147-150, 1979.

[2] C. F. Dagano, F. Bouthelier, and Y. Sheffi, "Multinomial probit and qualitative choice: a computationally efficient algorithm," Transportation Science, vol. 11, no. 4, pp. 338-358, 1977.

[3] H. Spiess and M. Florian, "Optimal strategies: a new assignment model for transit networks," Transportation Research Part B, vol. 23, no. 2, pp. 83-102, 1989. 
[4] J. de Cea and E. Fernandez, "Transit assignment for congested public transport systems: an equilibrium model," Transportation Science, vol. 27, no. 2, pp. 133-147, 1993.

[5] W. U. Jiahao, M. Florian, and P. Marcotte, "Transit equilibrium assignment: a model and solution algorithms," Transportation Science, vol. 128, no. 3, pp. 193-203, 1994.

[6] R. Fernández and R. Planzer, "On the capacity of bus transit systems," Transport Reviews, vol. 22, no. 3, pp. 267-293, 2002.

[7] M. G. H. Bell, J. D. Schmöcker, Y. Iida, and W. H. K. Lam, "Transit assignment: an application of absorbing Markov chains," in Transportation and Traffic Theory in the 21st Century, M. A. P. Taylor, Ed., pp. 43-62, 2002.

[8] C. Billi, G. Gentile, S. Nguyen, and S. Pallottino, "Rethinking the wait model at transit stops," in Proceedings of TRISTAN-Workshop, Guadelupe, 2004.

[9] M. Cepeda, R. Cominetti, and M. Florian, "A frequency-based assignment model for congested transit networks with strict capacity constraints: Characterization and computation of equilibria," Transportation Research Part B: Methodological, vol. 40, no. 6, pp. 437-459, 2006.

[10] G. Gentile, S. Nguyen, and S. Pallottino, "Route choice on transit networks with online information at stops," Transportation Science, vol. 39, no. 3, pp. 289-297, 2005.

[11] F. Kurauchi, M. G. H. Bell, and J.-D. Schmöcker, "Capacity constrained transit assignment with common lines," Journal of Mathematical Modelling and Algorithms, vol. 2, no. 4, pp. 309-327, 2003.

[12] H. Larrain and J. C. Muñoz, "Public transit corridor assignment assuming congestion due to passenger boarding and alighting," Networks and Spatial Economics, vol. 8, no. 2-3, pp. 241-256, 2008.

[13] G. Desaulniers and M. D. Hickman, "Public transit," in Handbooks in Operations Research and Management Science, vol. 14, chapter 2, pp. 69-12, 2007.

[14] J. D. Schmöcker, M. G. H. Bell, and F. Kurauchi, "A quasi-dynamic capacity constrained frequencybased transit assignment model," Transportation Research Part B, vol. 42, no. 10, pp. 925-945, 2008.

[15] A. Nuzzolo, U. Crisalli, and L. Rosati, "A schedule-based assignment model with explicit capacity constraints for congested transit networks," Transportation Research Part C, 2011.

[16] H. Spiess and M. Florian, "Optimal strategies: A new assignment model for transit networks," Transportation Research Part B, vol. 23, no. 2, pp. 83-102, 1989.

[17] Y. Sheffi, Urban Transportation Networks, Prentice-Hall, Englewood Cliffs, NJ, USA, 1984.

[18] R. B. Dial, "A probabilistic multipath traffic assignment model which obviates path enumeration," Transportation Research, vol. 5, no. 2, pp. 83-111, 1971.

[19] W. Wei, "Urban public transit system planning and manage technique," China Science press, pp. 93-94, 2002.

[20] Q. Xuyan, L. Huapu, and W. Yuanyuan, "A K-shortest-paths-based algorithm for stochastic traffic assignment model and comparison of computation precision with existing methods," in Proceedings of the Eastern Asia Society for Transportation Studies, vol. 5, pp. 1218-1232, 2005. 


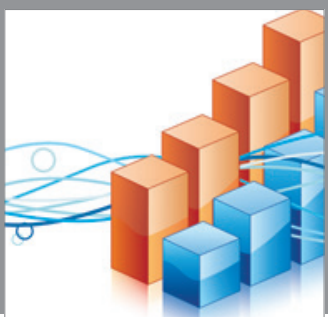

Advances in

Operations Research

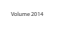

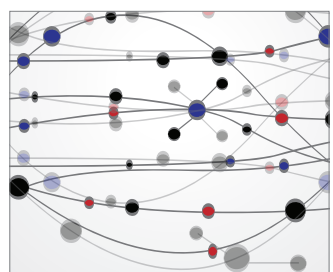

\section{The Scientific} World Journal
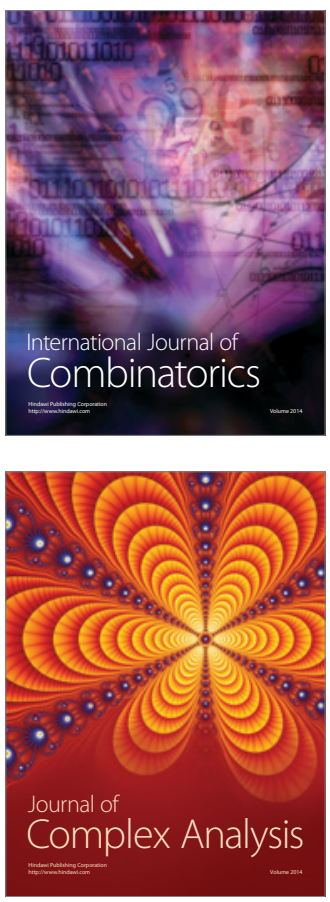

International Journal of

Mathematics and

Mathematical

Sciences
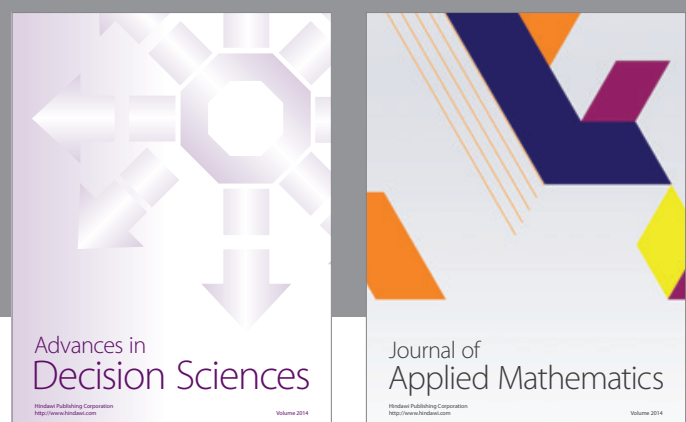

Journal of

Applied Mathematics
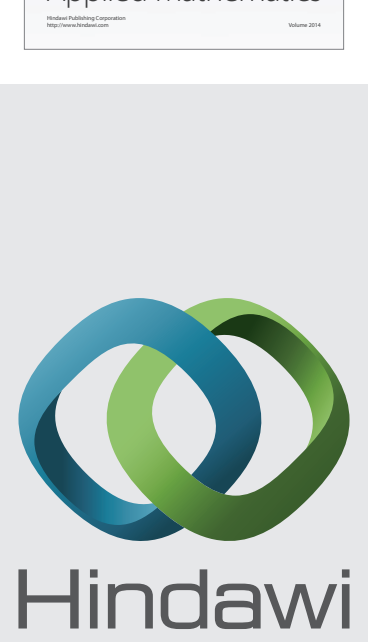

Submit your manuscripts at http://www.hindawi.com
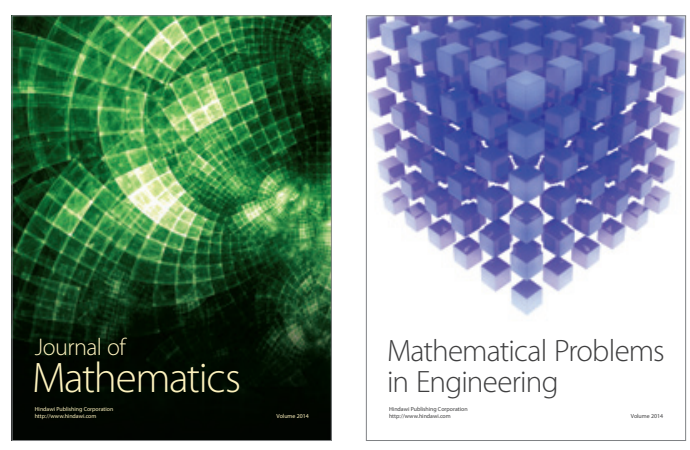

Mathematical Problems in Engineering
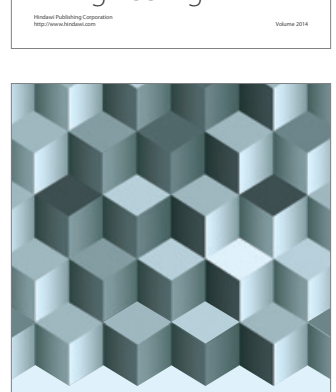

Journal of

Function Spaces
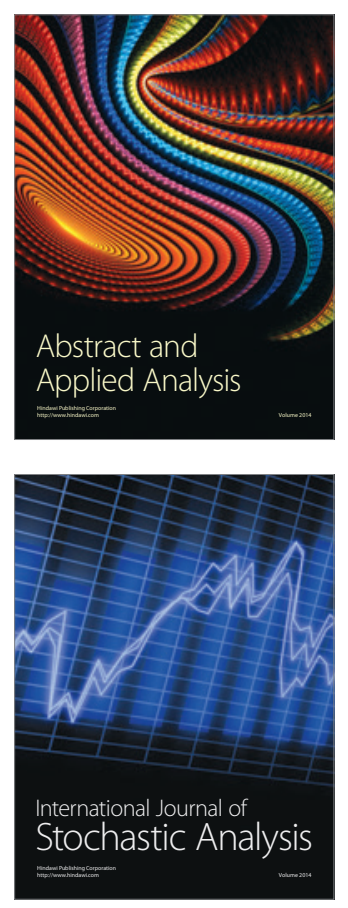

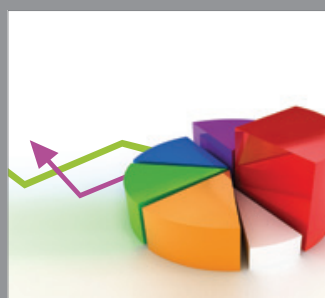

ournal of

Probability and Statistics

Promensencen
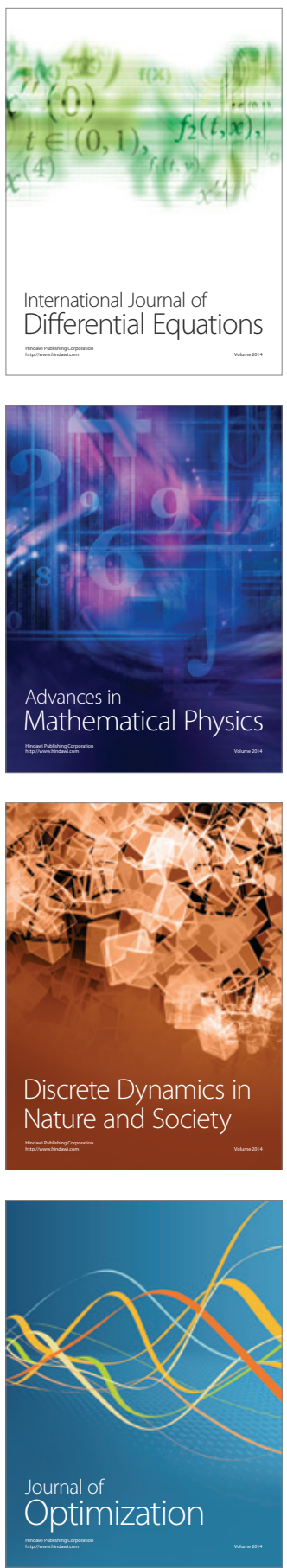